Vietnam Journal of Mechanics, VAST, Vol.42, No. 1 (2020), pp. 15-27

DOI: https://doi.org/10.15625/0866-7136/14397

\title{
EXPERIMENTAL AND NUMERICAL INVESTIGATIONS OF FULL-FIELD STRAIN MEASUREMENT AND FRACTURE PARAMETER OF LEAD-FREE SOLDER USING DIC TECHNIQUE
}

\author{
Tao Quang Bang ${ }^{1, *}$, Nguyen Van Thien $\mathrm{An}^{1}$, Lahouari Benabou ${ }^{2}$, Nguyen Xuan Hung ${ }^{3}$ \\ ${ }^{1}$ Danang University of Technology, University of Danang, Vietnam \\ ${ }^{2}$ LISV, University of Versailles Saint Quentin en Yvelines, University of Paris Saclay, France \\ ${ }^{3}$ CIRTech Institute, Ho Chi Minh City University of Technology (HUTECH), Vietnam \\ E-mail: tqbang@dut.udn.vn
}

Received: 05 September 2019 / Published online: 26 March 2020

\begin{abstract}
In this study, rupture of notched SENT specimens fabricated from a novel leadfree solder alloy is investigated. The lead-free solder alloy, focused on in this study, is particularly used as interconnect material in power modules of electric vehicles. Its commercial denomination is InnoLot and it can be used in harsh environments thanks to its improved reliability. Up to now, studies on their resistance to rupture remain relatively limited. Yet the comprehension of fracture behavior is essential for the correct design of the electronic packages which must be robust against fatigue and vibrations loads. The tests are performed with the help of a micro-tensile testing machine equipped with an optical system for full-field measurements with Digital Image Correlation. The images are taken at successive steps of deformation and the displacement field is measured in a region of interest which is the singularity dominated zone surrounding the plastic zone at the crack tip. The procedure consists then in comparing the measured field with the theoretical field given by the Williams' solution. The stress intensity factor is calculated by fitting the analytical fields to the experimental data. The effects of the size and shape of the zone of data collection, as well as that of the number of terms considered in the Williams's expansion series, are examined in the study. A method is also proposed for the automatic crack tip detection. From these finding, it is easy to predict the crack propagation and failure mechanism of solder joint. In addition, the theoretical solution of displacement, given by the Williams series, is compared with measurements to identify the coefficients of these series, including the stress intensity factor. Finally, a 5-order truncation of the Williams series seems sufficient to obtain a correct estimate of the stress intensity factor.
\end{abstract}

Keywords: lead-free solder, digital image correlation, plastic zone, fracture.

\section{INTRODUCTION}

Due to the RoHS and WEEE legislations for restricting the use of six hazardous materials in the manufacture of various types of electronic and electrical equipment, developing novel $\mathrm{Pb}$-free solders becomes a real challenge for many industrials in recent years.

(C) 2020 Vietnam Academy of Science and Technology 
Thanks to that, electronics manufacturers are developed a new generation of solder alloys without lead element that could maintain or improve the reliability of the solders. Indeed, many authors have investigated on the reliability of tin-silver-copper solders with small additions of some elements such as $\mathrm{Bi}, \mathrm{Zn}, \mathrm{Ni}, \mathrm{Al}, \mathrm{Sb}, \ldots$ [1-5]. The behaviors of these solders have been found to be particularly dependent on the amount of the reactive constituent element of the solders. Nevertheless, the trend of miniaturization of electronic products, as well as increasingly complex and harsh working conditions, brings huge challenge to their reliability. Thus, the reliability of electronic products receives wide attention. Furthermore, in electronic packaging, solder joint is one of the most easily failed parts, which has always been a hot research topic in this field. Indeed, from the literature, many researchers have focused on mechanical properties, failure mechanisms, and fracture parameters of solder joints.

In the fracture failure of the solder joints, crack growth is initiated in areas of high stress concentration such as areas with geometric accidents or defects inherent in the materials. In linear fracture mechanics, the stress intensity factor (SIF) is a parameter that makes it possible to quantify the criticality of a crack since it reflects the effect of loading on the evolution of the crack. Similarly, the displacement and stress fields can be characterized by the stress intensity factor. Therefore, this knowledge is important to prevent the brittle fracture that can occur in the case of pre-existing cracks. Many conventional experimental techniques, based on the use of strain gauges or Moiré Interferometric, allow determining the stress intensity factor. However, strain gauges only access a very limited number of measurements. Also, optical methods provide a complete field measurement, but they still remain difficult to implement and are not suitable for opaque materials. In comparison, the correlation of digital images proves to be a more efficient technique since it not only used with all types of materials but also at different scales (from the scale of the microstructure to that of the structure). The digital image correlation (DIC) technique is nowadays largely adopted as a reliable, non-destructive, low-cost technique to measure real-time local displacement on a flat surface of the specimen [6,7]. With the development of full-field measurement technique, it has been possible to analyze crack propagation experimentally with an increasing level of robustness.

Therefore, in this work, Digital Image Correlation method is used to evaluate stress intensity factor in notched specimens made from InnoLot solder alloy (SAC387-3Bi-1.5Sb$0.15 \mathrm{Ni}$ ). Notched SENT specimens fabricated from the InnoLot lead-free solder alloy is investigated. The tests are performed with the help of a micro-tensile testing machine equipped with an optical system for full-field measurements with Digital Image Correlation (DIC). The images are taken at successive steps of deformation and the displacement field is measured in a region of interest which is the singularity dominated zone surrounding the plastic zone at the crack tip. The procedure consists then in comparing the measured field with the theoretical field given by the Williams' solution. The stress intensity factor is calculated by fitting the analytical fields to the experimental data. The effects of the size and shape of the zone of data collection, as well as that of the number of terms considered in the Williams's expansion series, are examined in the study. Another factor that determines the accuracy of this method is the ability to correctly determine 
the position of the crack tip. This question is therefore also addressed in this work by proposing an algorithm for automatic detection of the crack at each new loading step.

\section{METHODOLOGY OF 2-D DIC}

The digital image correlation (DIC) method refers to an optical and non-contact measurement technique, which includes the steps of image acquisition, storage, and correlation since the 1980s [8-10]. It generates the full-field deformation, motion, and profile in all the directions. Benefiting from the enhancement of pixel amount, DIC method evolved to be a technique for the in-situ mechanics test with high sensitivity.

The in-plane 2-D DIC derives from the concept that camera traces the feature movement on the sample's surface. The camera sensor focuses on the planar object surface to record the images of the specimen under different loads. The specimen was painted or sprayed with black and white patterns as the features to give each pixel a grey value that ranges between 0 (black) and 255 (white). The light sources are important to illuminate the white and black features on the sample surface. In addition, the basic calculation unit is subset, which consists of several pixels. By grouping many pixels into one subset, it is capable of correlating and recognizing the shape and displacement of every subset. The shear deformation can be justified through the subset shape comparison. 2-D DIC achieves a 0.02 pixel measurement accuracy.

The technique of digital image correlation consists in comparing the deformed images of the surface of the sample with the reference image obtained before deformation. The correlation algorithm requires the surface images be "textured", which is obtained by speckling method. The sample was painted using an airbrush with diameter a nozzle of $0.2 \mathrm{~mm}$. This type of device is adapted to generate on small samples a sufficiently fine texture (sub-millimetric size paint stains).

The reference image is subdivided into subsets whose positions are determined on the deformed images. Consider a subset centered on the $P\left(x_{0}, y_{0}\right)$ point in the reference image (see Fig. 1(a)). A point $Q\left(x_{i}, y_{i}\right)$ in this subset becomes after deformation the point $Q^{\prime}\left(x_{i}^{\prime}, y_{i}^{\prime}\right)$ in the target subset by the following transformation

$$
\begin{gathered}
x_{i}^{\prime}=x_{i}+u+\frac{\partial u}{\partial x} \Delta x+\frac{\partial u}{\partial y} \Delta y, \\
y_{i}^{\prime}=y_{i}+v+\frac{\partial v}{\partial x} \Delta x+\frac{\partial v}{\partial y} \Delta y,
\end{gathered}
$$

where $u$ and $v$ are the components of the displacement of the center of the subset of reference $P\left(x_{0}, y_{0}\right) ; \frac{\partial u}{\partial x}, \frac{\partial u}{\partial y}, \frac{\partial v}{\partial x}, \frac{\partial v}{\partial y}$ are the gradients of displacement; $\Delta x=x_{i}-x_{0}, \Delta y=$ $y_{i}-y_{0}$.

To estimate the degree of similarity between the reference subset and the deformed subset, a correlation coefficient is calculated according to a specific criterion from the set of points of the subset. By searching for the extreme value of this coefficient, the displacement of the point $P$ can be determined.

Under the assumption of linear elastic elasticity of fracture, Williams [11] proposed a solution for displacement and stress fields near the crack front in the form of series 


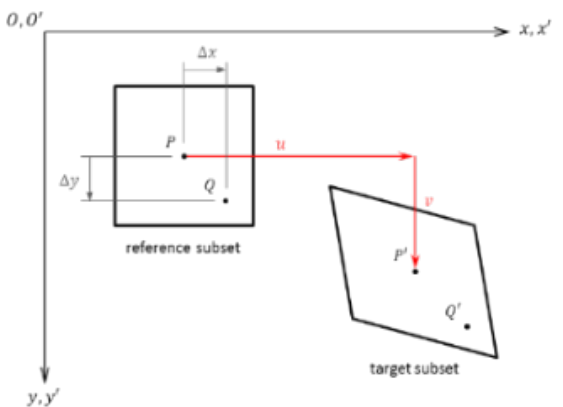

(a)

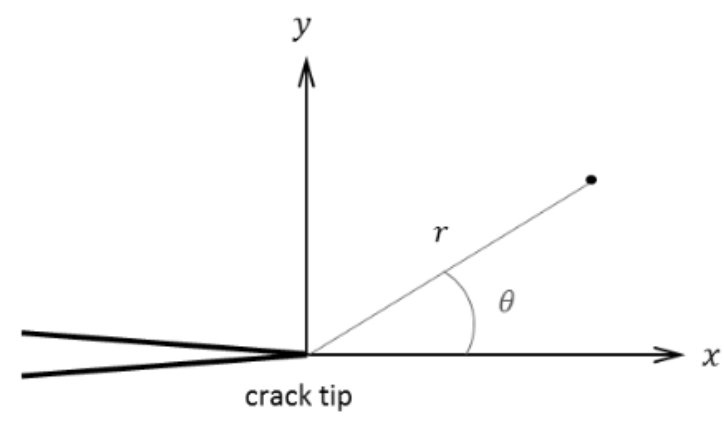

(b)

Fig. 1. Concept of the DIC based on the follow-up of a correlation window (subset) between the reference image and the deformed image of the surface (a), local coordinate system around the crack tip (b)

developments. In the case of a plane problem (see Fig. 1(b)), the field of displacement around a crack, embedded in a homogeneous isotropic medium and subjected to mode I, is given by

$$
\left\{\begin{array}{l}
u \\
v
\end{array}\right\}=\sum_{n=1}^{\infty} \frac{r^{n / 2}}{2 \mu} A_{n}\left\{\begin{array}{l}
\left(\kappa+\frac{n}{2}+(-1)^{n}\right) \cos \frac{n \theta}{2}-\frac{n}{2} \cos \left(\frac{n}{2}-2\right) \theta \\
\left(\kappa-\frac{n}{2}-(-1)^{n}\right) \sin \frac{n \theta}{2}+\frac{n}{2} \sin \left(\frac{n}{2}-2\right) \theta
\end{array}\right\},
$$

where $\mu$ is the shear modulus; $\kappa=3-4 v$ is in plane strain condition and $\kappa=(3-$ $v) /(1+v)$ in plane stress condition; $v$ is the Poisson's ratio and $r$ and $\theta$ are the polar coordinates of a point measured with respect to the crack tip.

The coefficients $A_{n}$ in Williams series depend on the geometric parameter $a / W$ (where $a$ is the crack length and $W$ is the width of the specimen). In particular, the first coefficient is linked to the stress intensity factor in Mode I, $A_{1}=K_{I} / \sqrt{2 \pi}$, and the second coefficient to "T-stress", $A_{2}=\sigma_{o x} / 4$. The higher orders reflect the influence of the boundary conditions in the case of a non-infinite medium. The displacements in Eq. (2) can be rewritten as

$$
u=\sum_{n=1}^{\infty} A_{n} f_{\text {In }}(r, \theta) \text { and } v=\sum_{n=1}^{\infty} A_{n} g_{I n}(r, \theta),
$$

where $f_{I n}(r, \theta)$ and $g_{I n}(r, \theta)$ are known functions depending on the polar coordinates.

By considering a possible rigid body movement accompanying the deformation and by truncating the series to order $N$, the displacement field expressed at the point of coordinates $\left(r_{k}, \theta_{k}\right)$ becomes

$$
\begin{aligned}
& u_{k}=\sum_{n=1}^{N} A_{n} f_{\text {In }}\left(r_{k}, \theta_{k}\right)+T_{x}-R y_{k}, \\
& v_{k}=\sum_{n=1}^{N} A_{n} g_{\text {In }}\left(r_{k}, \theta_{k}\right)+T_{y}+R x_{k},
\end{aligned}
$$


where $T_{x}$ and $T_{y}$ represent the components of the translation in the directions $x$ and $y$, and $R$ is the rotation.

\section{EXPRIMENTAL PROCEDURES}

\subsection{Experimental Procedure}

Samples of commercial 91.07Sn3.8Ag0.7C-3Bi-1.5Sb-0.15Ni solder alloy (thereby called SAC387-3Bi-1.5Sb-0.15Ni or InnoLot) were used in this study. The solder has begun to come into use in the packaging of some microelectronics components and devices, especially in car industry. Some previous studies have demonstrated that the mechanical properties like thermal cycling, fatigue life of InnoLot solder have improved by adding some elements $\mathrm{Ni}, \mathrm{Bi}$ and $\mathrm{Sb}[4,5]$.
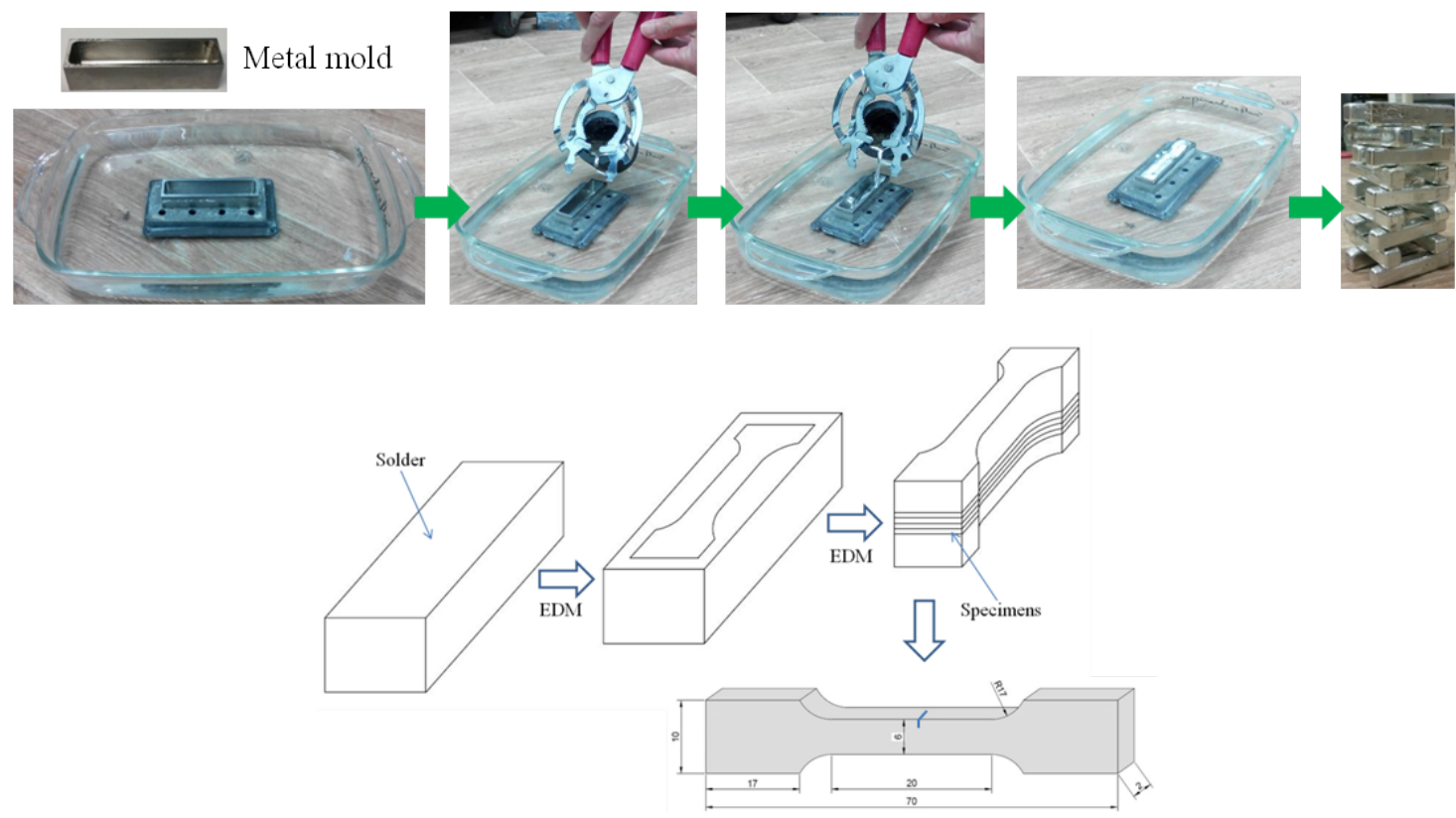

Fig. 2. The procedure for fabricating the SENT specimens and their dimensions (mm)

In this study, in order to conduct crack testing, bulk Single Edge Notched Tension (SENT) specimens were fabricated using the InnoLot solder material according to the following steps (see Fig. 2):

- The InnoLot solder material was melted in oven at $100^{\circ} \mathrm{C}$ above the liquidus point (around $330^{\circ} \mathrm{C}$ ) in a graphite cup and then the melted solder alloy was quickly poured into a metal mold which was made from 304-Inox material with outer dimensions of $16 \mathrm{~mm}$ width, $18 \mathrm{~mm}$ height and $80 \mathrm{~mm}$ length. The metal mold was then placed into water with temperature in the range of $25^{\circ} \mathrm{C}$ to $35^{\circ} \mathrm{C}$ based on recommendation of manufacturers;

- The cooling process is maintained until solidification (over 3 minutes after pouring), after that the solid solder block is pulled out from the mold; 
- The solder blocks are then machined and sliced by Electrical Discharge Machining (EDM) into standard flat dog-bone specimens with pre-crack. The specimens have a gauge length of $20 \mathrm{~mm}$, a fillet radius of $17 \mathrm{~mm}$ to prevent any stress concentration due to sharp corners, and a rectangular cross section of $2.0 \times 6.0 \mathrm{~mm}^{2}$ in the central part.

- Finally, before testing, the specimens are annealed in an oven at $100^{\circ} \mathrm{C}$ for $120 \mathrm{~min}$ utes and then cooled down to room temperature in the chamber to stabilize microstructure and remove any residual stresses induced by the cutting process.

The DIC technique consists in comparing images of the deformed surface of a specimen with a reference image captured before loading. The algorithm necessitates that a speckle pattern exists on the analysed surface, which is obtained by applying a white paint, followed by a black over-sprayed paint using an airbrush system [12]. The opensource subset-based 2D program Ncorr has been used to process the DIC data [13]. The package is implemented in MATLAB, which allows us to get quite straightforwardly the measured fields and add code for the computation of the fracture parameters. As regards the mechanical testing of the small notched specimens, it is carried out with a miniaturized tensile machine with a suited loading capacity and a wide range of crosshead speeds [14]. It is equipped with a tension/compression load cell of $2 \mathrm{kN}$ capacity, a Linear Variable Differential Transformer (LVDT) of $\pm 6 \mathrm{~mm}$ measurement range, and a stepper motor associated with a high-resolution micro-stepping driver for the control of the crosshead motion. A micro-tensile machine was designed that equipped with DIC system as shown in Fig. 3.

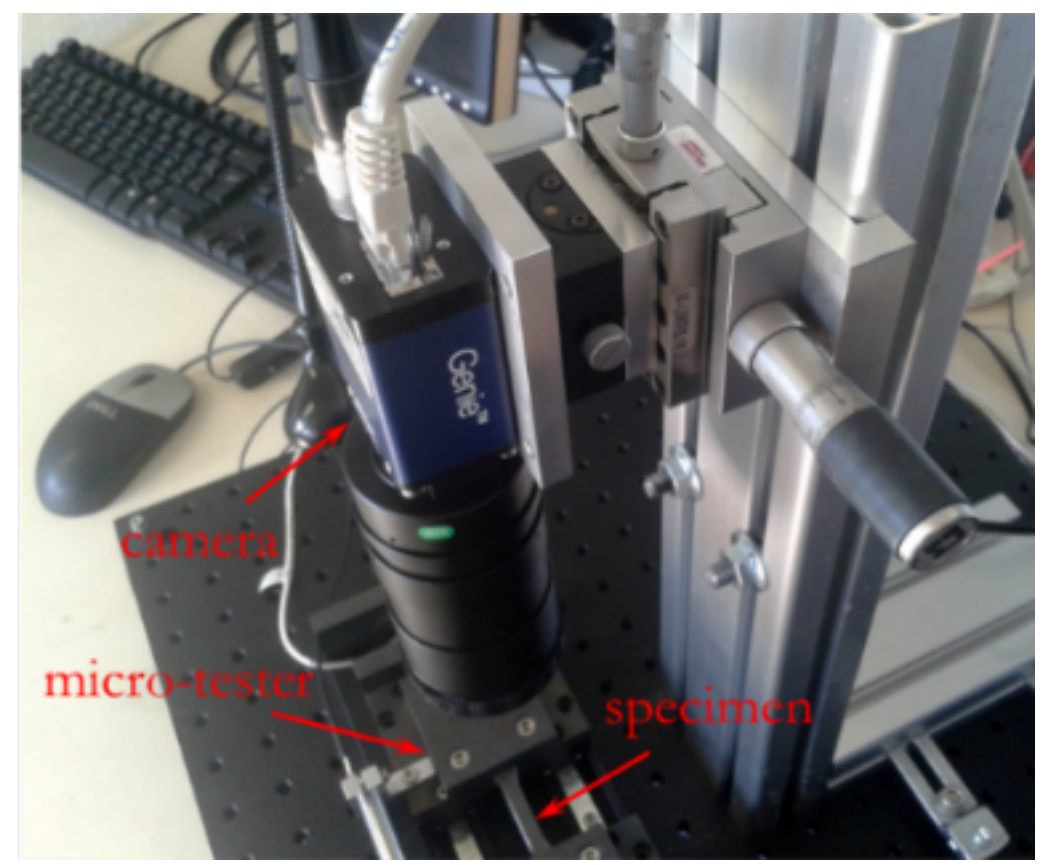

Fig. 3. A micro tension equipped with digital image correlation measurement for DIC testing 


\subsection{Determination of the parameters of rupture}

The DIC technique measures the displacements of a set of points in a region at the vicinity of the crack. The terms of the Williams series are then determined by performing a least squares minimization of the difference between the measured displacements and the analytical solutions [11]. Since the latter are based on the hypotheses of the linear elastic mechanism of the fracture, the points must be taken in the singular elastic zone surrounding the plasticized zone at the crack tip. The size of the plasticized zone can be estimated using the relationship

$$
r_{p}=\alpha\left(K_{I} / \sigma_{y}\right)^{2}
$$

where $\alpha=1 / \pi$ for the Irwin approximation (plane stresses); and $\alpha=\pi / 8$ for the Dugdale approximation.

Consider the displacement measurements of selected points $M$ in the sampling area. An over determined system is obtained by taking a number of equations greater than the number of unknowns, i.e. $2 M \geq N+1$ where $N$ and the number of coefficients to be determined in the Williams series. Eq. (3) can then be rewritten for points $M$ in a matrix form

$$
\begin{aligned}
& \left\{\begin{array}{c}
u_{1} \\
\vdots \\
u_{M} \\
v_{1} \\
\vdots \\
v_{M}
\end{array}\right\}=\left[\begin{array}{cccccc}
f_{I 1}\left(r_{1}, \theta_{1}\right) & \cdots & f_{I N}\left(r_{1}, \theta_{1}\right) & 1 & 0 & -r_{1} \sin \theta_{1} \\
\vdots & \ddots & \vdots & \vdots & \vdots & \vdots \\
f_{I 1}\left(r_{M}, \theta_{M}\right) & \cdots & f_{I N}\left(r_{M}, \theta_{M}\right) & 1 & 0 & -r_{M} \sin \theta_{M} \\
g_{I 1}\left(r_{1}, \theta_{1}\right) & \cdots & g_{I N}\left(r_{1}, \theta_{1}\right) & 0 & 1 & r_{1} \cos \theta_{1} \\
\vdots & \ddots & \vdots & \vdots & \vdots & \vdots \\
g_{I 1}\left(r_{M}, \theta_{M}\right) & \cdots & g_{I N}\left(r_{M}, \theta_{M}\right) & 0 & 1 & r_{M} \cos \theta_{M}
\end{array}\right]\left\{\begin{array}{c}
A_{1} \\
\vdots \\
A_{N} \\
T_{x} \\
T_{y} \\
R
\end{array}\right\} \\
& \text { or }\{X\}=\left([\mathrm{B}]^{T}[B]\right)^{-1}[B]^{T}\{U\} .
\end{aligned}
$$

If the crack tip location is known, a set of linear equations is obtained, whose solution can be expressed in the least-squares sense as $\{X\}=\left([B]^{T}[B]\right)^{-1}[B]^{T}\{U\}$. In order to use the so-called over-deterministic method (ODM), the number of equations corresponding to the number of points considered for data collection must be greater than the number of unknowns, i.e. the number of the expansion terms that has to be calculated.

Determining accurately the location of the crack tip from images obtained experimentally, like in DIC measurements, is required for a good quality fit of the field equations to the experimental data. A method proposed by [15] is to determine the stress intensity factor by finding the location of crack tip which minimizes the error in least squares by trial and errors. Such an optimization method, which is based on the fundamentals of LEFM, has been widely utilized for characterizing failure but is limited to fully elastic fields, and requires accurate location of the crack tip. In contrast to the optimization method, integral methods can be applied to both elastic and elastoplastic fields. 


\section{NUMERICAL IMPLEMENTATION}

The identification procedure is implemented in the Matlab software. The latter uses for each loading step the data extracted from the DIC analysis made with the Ncorr software [13], in particular the coordinates and displacements at the points of the sampling zone. The influence of the shape of this area is studied by working with two different geometries: rectangular and annular (Fig. 4). The experimental values of the displacements at the different points of the grid are then compared iteratively with the theoretical values given by Eq. (2) to determine the coefficients of the Williams series minimizing the residue.

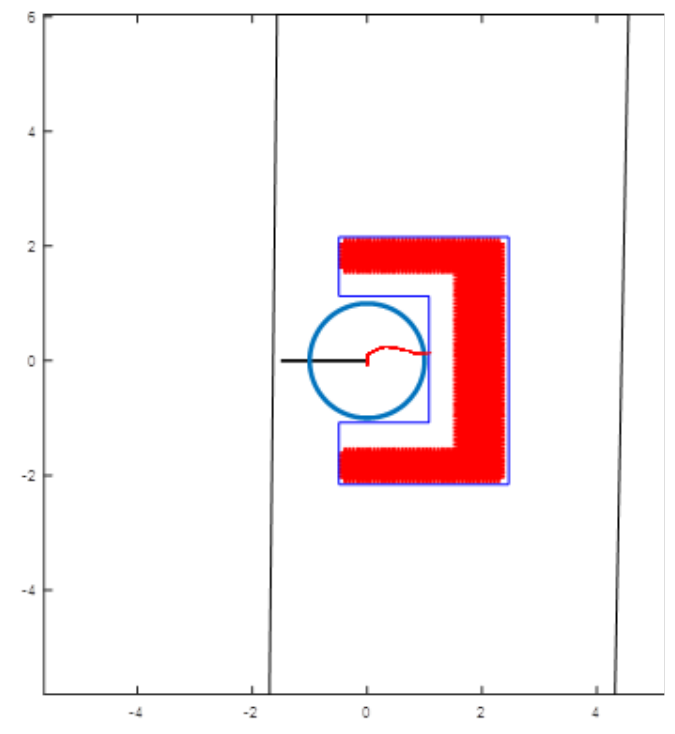

(a) Rectangular case

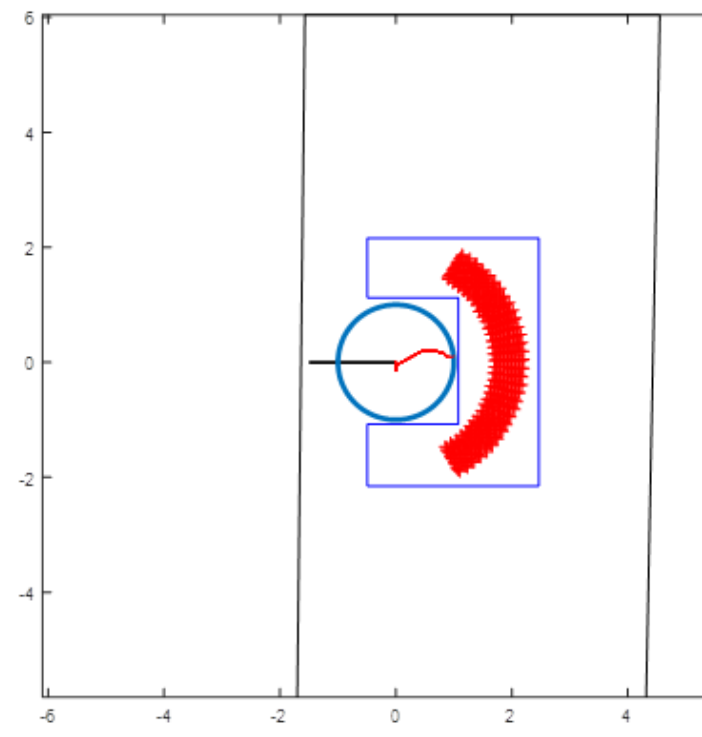

(b) Annular case

Fig. 4. Form of the displacement data sampling zone (contained in the singular elastic region)

Another problem to be solved in the numerical implementation concerns the knowledge of the position of the crack front must be sufficiently precise to make the correct adjustment of the parameters of the analytical solution. A method of automatically locating the crack front consists of performing the identification procedure for different assumed positions of the crack front, taken in the vicinity of a roughly estimated initial position. The position leading to the minimum error in calculating the difference between the measured field and the analytical field will be considered as the true position of the crack.

\section{RESULTS}

\subsection{Determination of crack evolution in a SENT specimen}

Fig. 5 shows full-field strain measurement, deformation profile and evolution of crack length of SENT specimens using DIC technique, respectively. In fracture toughness 
experiments, the crack length has to be measured with enough accuracy during its propagation. Using digital images acquired in the course of deformation is a straightforward way to measure the crack length evolution. However, with such a manual method, some uncertainty is generated in the measurements since these latter depend on the accuracy with which the user identifies the crack path on the gray scale images. The uncertainty becomes even more critical when the crack is not clearly visible and cannot be tracked correctly. By post-processing the images with the DIC, it has been found that the crack tip advance can be followed successfully [16].

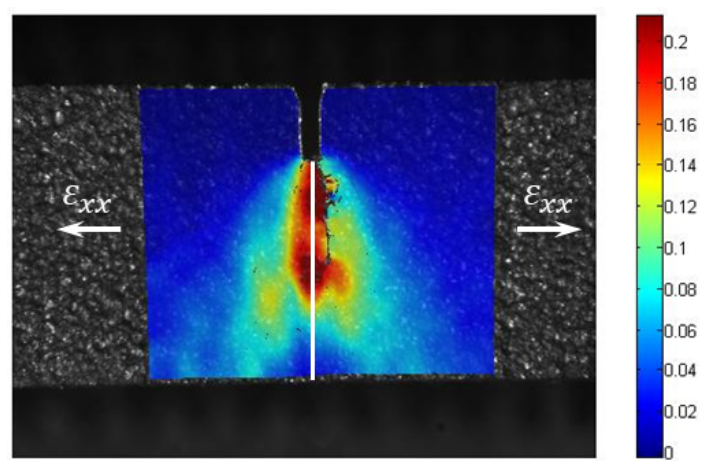

(a)

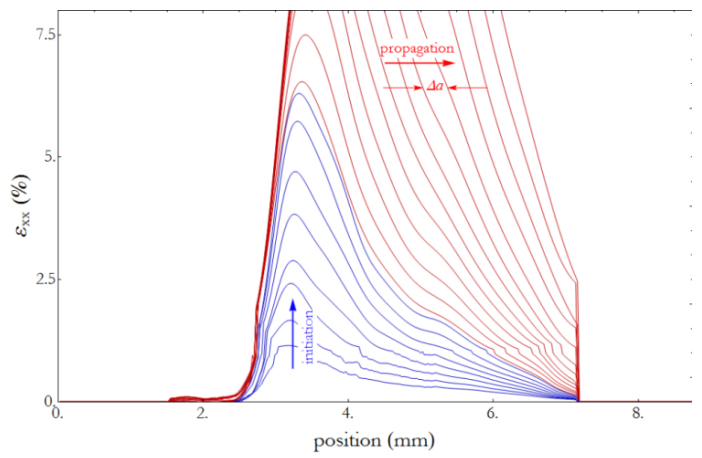

(b)

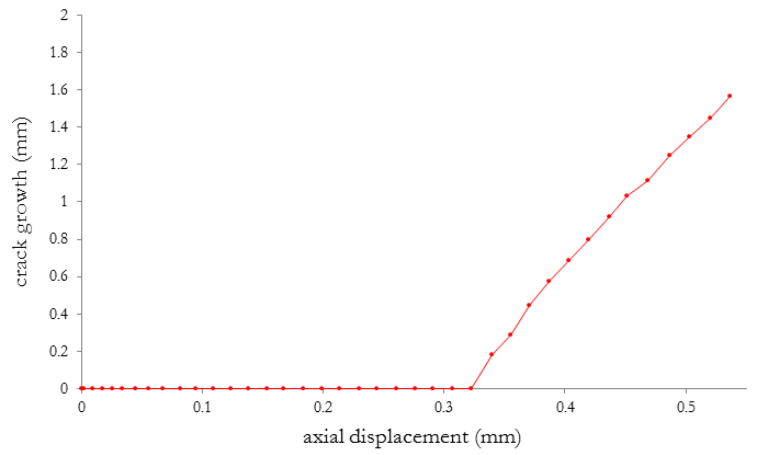

(c)

Fig. 5. Full-field strain measurement (a), deformation of profile (b) and evolution of crack length (c) for SENT specimen

DIC offers a means to monitor the strain ahead of the crack tip. Due to the stress concentration effect, the axial strain (in the direction of loading) is high near the crack tip and drops as a function of distance away from the crack tip. As an illustration, Fig. 5(b) shows the strain profiles along the crack line prior and after crack propagation in the case of a SENT specimen. These strain profiles are extracted in steps of $4 \mathrm{~s}$ over a period of $200 \mathrm{~s}$ from subsequent images in the course of loading (50 images in total). Detecting the absolute position of the crack tip is not straightforward, but, between two consecutive 
images, there is an increment in crack growth, corresponding exactly to the shift in the strain profile. To calculate this shift, there is a need to track a selected strain as it follows the crack across the specimen. There is in theory some uncertainty on this strain value but this should leave the offset unchanged. Fig. 5(c) shows the crack length-applied displacement curves for the notched specimen. Following the same procedure as in Reu et al. [16], the crack tip location is calibrated in this work with a characteristic strain surface corresponding to the maximum value of axial strain in the immediate vicinity of the notch at the onset of crack propagation.

\subsection{Fracture parameters}

Fig. 6 shows the evolution of the parameter $A_{1}$ (related to the stress intensity factor $K_{1}$ ) for a deformed image of the InnoLot solder specimen. An influence of the shape of the sampling zone (rectangular vs. annular) appears also in the results, with an estimation of the parameter $A_{1}$ higher in the annular case. Similarly, the truncation order of the Williams series has an influence since an adjustment made to the order 3 gives significantly lower values than the orders 5 and 7 which lead to similar estimates.

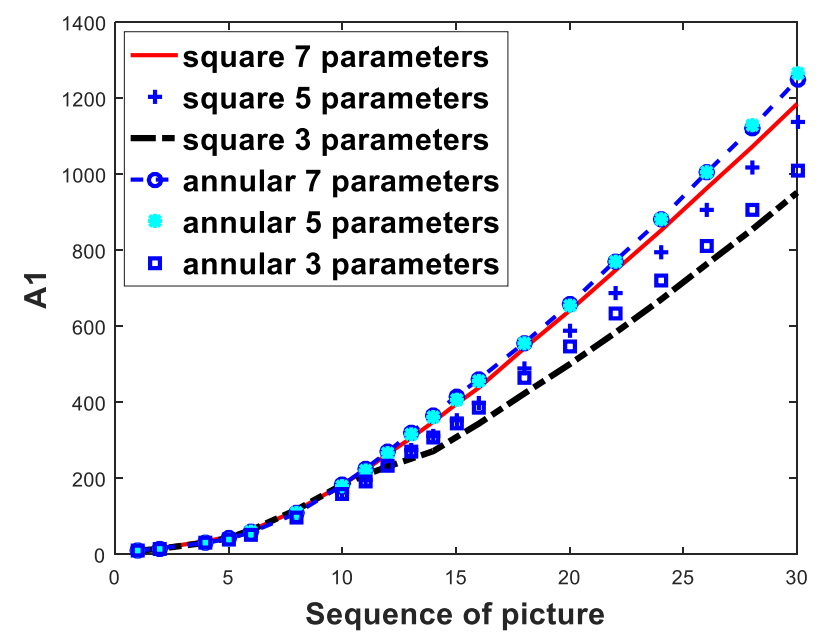

Fig. 6. Evolution of the factor during loading (influences of the shape of the sampling zone and the truncation order of Williams series)

Fig. 7 illustrates the progression, at different loading levels, of the crack as predicted by the crack-edge detection procedure. The adjustment error between the theoretical solution and the measurement is represented in the sampling zone (rectangular case) at each step. It shows that the accuracy of the proposed adjustment procedure is very satisfactory with an error remaining below $2.5 \%$. 

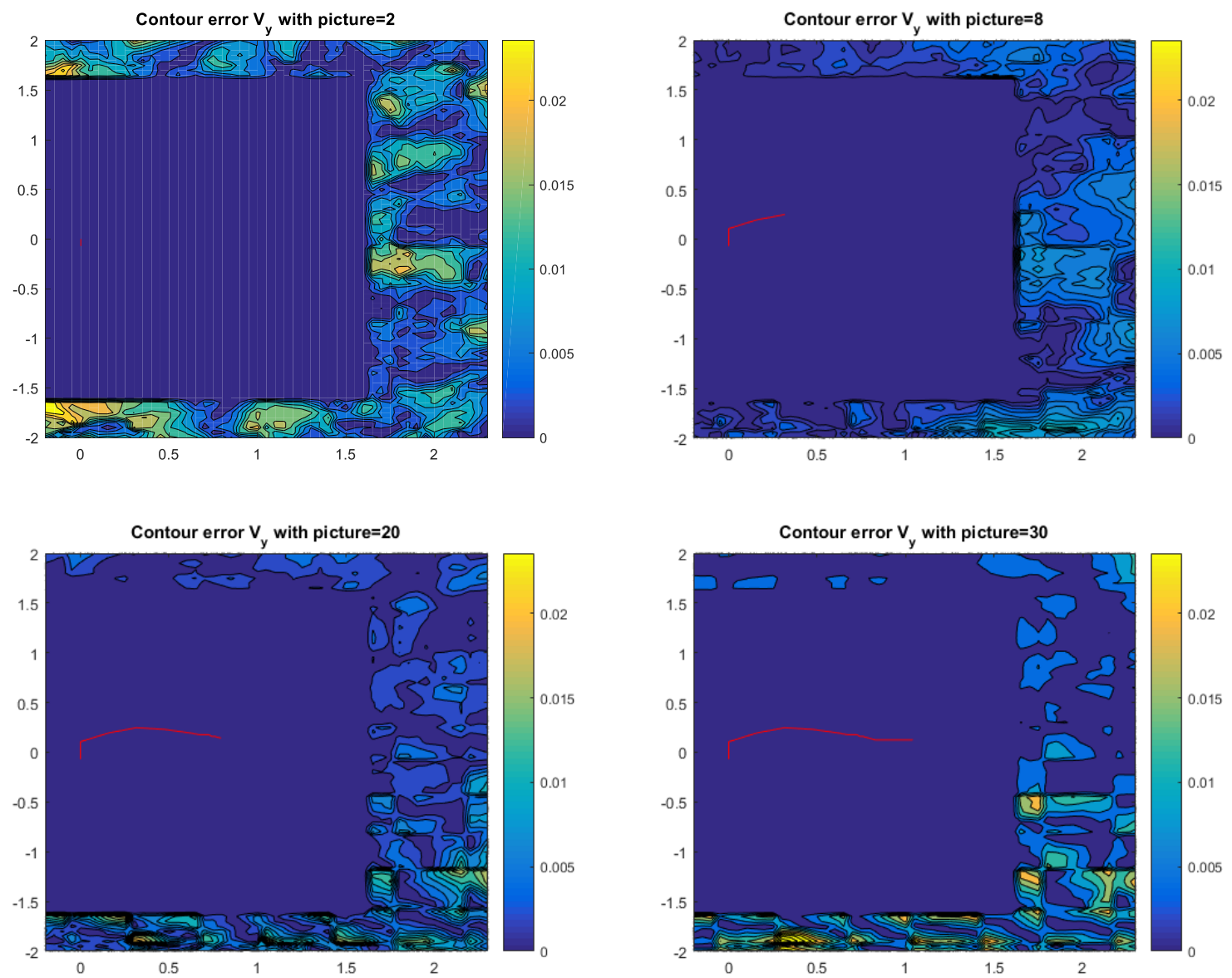

Fig. 7. Progression of the crack at different loading levels (images $2,8,20,30$, respectively) and representation of the adjustment error in the sampling zone

\section{CONCLUSIONS}

In this study, DIC technique has been used for SENT specimens made from a novel lead-free solder material. The procedure to fabricate the SENT specimens as well as experimental setup for cracking test has been also thoroughly described. A procedure for identifying fracture parameters for a lead-free solder has been investigated in this paper. The findings show that based on DIC technique the full-field strain measurement and crack propagation of SENT specimen have extracted. From these finding, it is easy to predict the crack propagation and failure mechanism of solder joint. In addition, the theoretical solution of displacement, given by the Williams series, is compared with measurements to identify the coefficients of these series, including the stress intensity factor. Since the accuracy of the results strongly depends on the knowledge of the position of the crack, a technique of automatic detection of the latter during loading has been integrated into the general procedure. The technique consists in retaining the position leading to 
the minimal adjustment error between predictions and measurements. The shape of the data collection area, including the singular elasticity region, is studied by considering the rectangular and annular cases. A form of the first type seems to lead to a smaller error. Finally, a 5-order truncation of the Williams series seems sufficient to obtain a correct estimate of the stress intensity factor.

\section{ACKNOWLEDGMENT}

This research is funded by Funds for Science and Technology Development of the University of Danang under project number B2018-DN02-38 and Vietnam National Foundation for Science and Technology Development (NAFOSTED) under grant number $107.01-2018.32$.

\section{REFERENCES}

[1] L. Zhang, S.-b. Xue, L.-1. Gao, G. Zeng, Z. Sheng, Y. Chen, and S.-l. Yu. Effects of rare earths on properties and microstructures of lead-free solder alloys. Journal of Materials Science: Materials in Electronics, 20, (8), (2009), pp. 685-694. https://doi.org/10.1007/s10854-009-9895-2.

[2] A. E. Hammad. Evolution of microstructure, thermal and creep properties of Ni-doped Sn$0.5 \mathrm{Ag}-0.7 \mathrm{Cu}$ low-Ag solder alloys for electronic applications. Materials $\mathcal{E}$ Design, 52, (2013), pp. 663-670. https://doi.org/10.1016/j.matdes.2013.05.102.

[3] M. H. Mahdavifard, M. F. M. Sabri, D. A. Shnawah, S. M. Said, I. A. Badruddin, and S. Rozali. The effect of iron and bismuth addition on the microstructural, mechanical, and thermal properties of Sn-1Ag-0.5 Cu solder alloy. Microelectronics Reliability, 55, (9-10), (2015), pp. 1886-1890. https://doi.org/10.1016/j.microrel.2015.06.134.

[4] Q. B. Tao, L. Benabou, V. N. Le, H. Hwang, and D. B. Luu. Viscoplastic characterization and post-rupture microanalysis of a novel lead-free solder with small additions of $\mathrm{Bi}, \mathrm{Sb}$ and Ni. Journal of Alloys and Compounds, 694, (2017), pp. 892-904. https://doi.org/10.1016/j.jallcom.2016.10.025.

[5] Q. B. Tao, L. Benabou, L. Vivet, V. N. Le, and F. B. Ouezdou. Effect of $\mathrm{Ni}$ and Sb additions and testing conditions on the mechanical properties and microstructures of lead-free solder joints. Materials Science and Engineering: A, 669, (2016), pp. 403-416. https://doi.org/10.1016/j.msea.2016.05.102.

[6] M. Mokhtari, P. Lopez-Crespo, B. Moreno, and M. Zanganeh. Some experimental observations of crack-tip mechanics with displacement data. Frattura ed Integrita Strutturale, 9, (33), (2015), pp. 143-150. https://doi.org/10.3221/igf-esis.33.18.

[7] F. Hild and S. Roux. Measuring stress intensity factors with a camera: Integrated digital image correlation (I-DIC). Comptes Rendus Mécanique, 334, (1), (2006), pp. 8-12. https://doi.org/10.1016/j.crme.2005.11.002.

[8] Y. Niu, H. Lee, and S. Park. A new in-situ warpage measurement of a wafer with specklefree digital image correlation (DIC) method. In 2015 IEEE 65th Electronic Components and Technology Conference (ECTC), (2015), pp. 425-431.

[9] M. A. Sutton, C. Mingqi, W. H. Peters, Y. J. Chao, and S. R. McNeill. Application of an optimized digital correlation method to planar deformation analysis. Image and Vision Computing, 4, (3), (1986), pp. 143-150. https://doi.org/10.1016/0262-8856(86)90057-0.

[10] M. A. Sutton, W. J. Wolters, W. H. Peters, W. F. Ranson, and S. R. McNeill. Determination of displacements using an improved digital correlation method. Image and Vision Computing, 1, (3), (1983), pp. 133-139. https://doi.org/10.1016/0262-8856(83)90064-1. 
[11] M. L. Williams. On the stress distribution at the base of a stationary crack. Journal of Applied Mechanics, 24, (1957), pp. 109-114.

[12] L. Benabou and Q. B. Tao. Development and first assessment of a DIC system for a microtensile tester used for solder characterization. Experimental Techniques, 41, (3), (2017), pp. 317326. https://doi.org/10.1007/s40799-017-0175-4.

[13] J. Blaber, B. Adair, and A. Antoniou. Ncorr: open-source 2D digital image correlation matlab software. Experimental Mechanics, 55, (6), (2015), pp. 1105-1122. https://doi.org/10.1007/s11340-015-0009-1.

[14] Q. B. Tao, L. Benabou, L. Vivet, K. L. Tan, J. M. Morelle, V. N. Le, and F. Ben Ouezdou. A design of a new miniature device for solder joints' mechanical properties evaluation. Proceedings of the Institution of Mechanical Engineers, Part C: Journal of Mechanical Engineering Science, 231, (20), (2017), pp. 3818-3830, https://doi.org/10.1177/0954406216654728.

[15] S. McNeill, W. Peters, and M. Sutton. Estimation of stress intensity factor by digital image correlation. Engineering Fracture Mechanics, 28, (1), (1987), pp. 101-112. https://doi.org/10.1016/0013-7944(87)90124-x.

[16] P. L. Reu, B. R. Rogillio, and G. W. Wellman. Crack tip growth measurement using digital image correlation. In Experimental Analysis of Nano and Engineering Materials and Structures, pp. 555-556. (2007). https://doi.org/10.1007/978-1-4020-6239-1_275. 